\title{
PERCEPÇÃO DE USUÁRIOS DE UMA UNIDADE BÁSICA DE SAÚDE SOBRE DOR CRÔNICA MUSCULOESQUELÉTICA E ESTRATÉGIAS DE GERENCIAMENTO DA DOR
}

\author{
PERCEPTION OF USERS OF A BASIC HEALTH UNIT ABOUT CHRONIC \\ MUSCULOSKELETAL PAIN AND PAIN MANAGEMENT STRATEGIES
}

\author{
Kácia Guedes de Oliveira ${ }^{1}$ \\ Álisson Nogueira Aquino ${ }^{2}$ \\ Débora Gomes da Silva Araújo ${ }^{3}$ \\ Maria Laura Lima da Silva ${ }^{4}$ \\ Thiago Bezerra dos Anjos ${ }^{5}$ \\ Tarcia Regina da Silva ${ }^{6}$ \\ Polyana Felipe Ferreira da Costa $^{7}$ \\ Rita di Cássia de Oliveira Angelo ${ }^{8}$
}

\begin{abstract}
Resumo: Abrangendo a dor como um fenômeno multifatorial e individual, este estudo com abordagem qualitativa, empregando a técnica de grupos focais, buscou ouvir e analisar as percepções, crenças e tomadas de decisão para autogerenciamento da dor de portadores de dor crônica musculoesquelética usuários da atenção básica à saúde. A partir da análise categorial temática os relatos de nove participantes foram agrupados em três categorias principais: "Percebendo a dor crônica e pensamentos associados"; "A dor atrapalha a saúde mental e o que costuma ser feito?" e "A virada: participação no projeto de educação em dor crônica". As sessões grupais favoreceram a reflexão sobre a condição dolorosa, o compartilhamento de sentimentos e o desenvolvimento de medidas de autocuidado. A escuta atenta contribui para maior autonomia e motiva o paciente a incorporar novas estratégias para lidar com a persistência da dor.
\end{abstract}

\footnotetext{
${ }^{1}$ Acadêmica do Curso de Bacharelado em Medicina, Universidade de Pernambuco (UPE), Serra Talhada, Pernambuco, Brasil.kacia.guedes@upe.br

${ }^{2}$ Acadêmico do Curso de Bacharelado em Medicina, Universidade de Pernambuco (UPE), Serra Talhada, Pernambuco, Brasil. alisson.aquino@upe.br

${ }^{3}$ Acadêmica do Curso de Bacharelado em Medicina, Universidade de Pernambuco (UPE), Serra Talhada, Pernambuco, Brasil. debora.gomesaraujo@upe.br

${ }^{4}$ Acadêmica do Curso de Bacharelado em Medicina, Universidade de Pernambuco (UPE), Serra Talhada, Pernambuco, Brasil. laura.lima@upe.br

${ }^{5}$ Acadêmico do Curso de Bacharelado em Medicina, Universidade de Pernambuco (UPE), Serra Talhada, Pernambuco, Brasil. thiago.bezerraa@upe.br

${ }^{6}$ Doutora em Educação, Universidade Federal da Paraíba (UFPB). Professora Adjunta do Programa de PósGraduação em Saúde e Desenvolvimento Socioambiental (PPGSDS) da Universidade de Pernambuco (UPE), Garanhuns, Pernambuco, Brasil. tarcia.silva@upe.br

${ }^{7}$ Mestre em Saúde Coletiva, Universidade Federal de Pernambuco (UFPE). Professora Assistente do Curso de Bacharelado em Medicina, Universidade de Pernambuco (UPE), Serra Talhada, Pernambuco, Brasil. polyana.costa@upe.br

${ }^{8}$ Doutora em Neuropsiquiatria e Ciências do Comportamento, Universidade Federal de Pernambuco (UFPE). Professora Adjunta do Programa de Pós-Graduação em Saúde e Desenvolvimento Socioambiental (PPGSDS) da Universidade de Pernambuco (UPE), Garanhuns, Pernambuco, Brasil. Professora Adjunta do Curso de Bacharelado em Medicina, Universidade de Pernambuco (UPE), Serra Talhada, Pernambuco, Brasil. rita.angelo@upe.br
} 
Palavras-chave: Dor crônica; Pesquisa Qualitativa; Atenção Primária à Saúde.

Abstract: Covering pain as a multifactorial and individual phenomenon, this study with a qualitative approach, using the technique of focus groups, sought to listen and analyze the perceptions, beliefs and decision-making for self-management of pain in patients with chronic musculoskeletal pain users of basic health care unit. From the thematic categorical analysis, the reports of nine participants were classified into three main categories: "Perceiving chronic pain and associated thoughts"; "Does pain hinder mental health and what is usually done?" and "The turning point: participation in the chronic pain education project". The group sessions favored reflection on the painful condition, the sharing of feelings and the development of self-care measures. Attentive listening contributes to greater autonomy and motivates patients to incorporate new strategies to deal with persistent pain.

Keywords: Chronic Pain; Qualitative research; Primary Health Care.

\section{Introdução}

A dor é um fenômeno multidimensional, inerente à existência humana, presente em todo o ciclo da vida (SOUZA et al., 2016). Pode estar presente em vários momentos cotidianos de forma breve ou persistente, sem necessariamente ter uma causa específica. A dor persistente ou crônica é definida como um desconforto que persiste contínua ou episodicamente por mais de 3 meses, possuindo majoritariamente origem musculoesquelética (SEMERU; HALIM, 2019). Essa condição de saúde acomete uma entre cinco pessoas na população mundial, está associada a efeitos negativos sobre a saúde mental (RODRIGUES et al., 2021) e é uma das condições de saúde mais onerosas em termos de anos vividos com incapacidade (JAMES et al., 2018).

Enquanto a dor aguda consiste em um sinal de alerta significativo, que surge como indicador de uma possível injúria tissular, com duração limitada no tempo e espaço e cessando com a resolução do evento nóxico (BOSCAROL et al., 2019); a dor crônica não tem finalidade biológica de alerta, possui caráter contínuo e duração prolongada, e pode ser influenciada por diversos fatores além do dano tissular. A persistência da dor ocasiona incapacidade por não responder positivamente aos tratamentos farmacológicos convencionais (AGUIAR; DUSSAN-SARRIA; SOUZA, 2019).

As dores crônicas de origem musculoesquelética (DCME) estão entre os tipos mais comuns de dores persistentes (MILLS; NICOLSON; SMITH, 2019), e acometem mundialmente cerca de 27 milhões de pessoas com faixa etária superior a 18 anos (MORAIS et al., 2019). Embora não seja possível afirmar com precisão a prevalência de dor crônica no Brasil (VASCONCELOS; ARAÚJO, 2018), estima-se um acometimento de $39 \%$ da população (SOUZA et al., 2017), configurando um relevante problema de saúde pública. 
Atualmente, a Associação Internacional para o Estudo da Dor considera que o impacto da dor na vida dos indivíduos seja influenciado por fatores biopsicossociais, reforçando a importância da subjetividade na percepção álgica (RAJA et al., 2020). De acordo com Mendes (2019), a experiência dolorosa é genuinamente humana, sendo vivenciada de forma singular e subjetiva por aquele que sente a dor, e suscita emoções e significados próprios.

A partir da compreensão de que a cronicidade da dor possui forte interferência na vida de seus portadores, já que se associa a alterações de personalidade, do estilo de vida e da capacidade funcional, a dor crônica deve ser caracterizada como uma doença (ARAÚJO, 2019); devendo sua abordagem ponderar os aspectos subjetivos de quem a sente, na medida em que cada paciente a expressa de forma diferente, mesmo que a sua origem seja semelhante (MENDES, 2019; MOURA et al., 2017; RAJA et al., 2020).

Segundo Moura et al., (2017), deve-se entender que o atendimento adequado ao portador de dor crônica exige uma avaliação precisa e criteriosa, não apenas da doença que pode estar causando a dor, mas também de uma infinidade de fatores comportamentais e psicossociais. Por conseguinte, torna-se essencial que os profissionais de saúde pratiquem a escuta ativa do paciente com dor, concentrando-se realmente no que está sendo dito, evitando avaliar e abordar a dor de forma pontual e isolada.

Apesar da atualização do conceito de dor e da aceitação de suas múltiplas subjetividades (RAJA et al., 2020), na prática clínica diária nas unidades de atenção primária à saúde, os pacientes ainda são tratados dentro do modelo biomédico, ou seja, são medicados e encaminhados a especialistas, que novamente prescrevem medicamentos (DE PAULA PRUDENTE et al., 2020). Em adição, diversas pesquisas atuais (AGUIAR; DUSSAN-SARRIA; SOUZA, 2019; DIONÍSIO; SALERMO; PADILHA, 2020; RODRIGUES et al., 2021) relacionadas ao tema se atém a dados quantitativos, não incluindo os aspectos qualitativos da dor crônica pela ótica de seus portadores.

Deste modo, ainda são escassos dados sobre crenças, percepções e significados que portadores de DCME, usuários de unidades básicas de saúde, atribuem às estratégias de intervenção direcionadas ao automanejo da dor (JESUS; SVENTNISCKAS; VIEIRA, 2019). Logo, considerando os aspectos multidimensionais envolvidos na dor crônica, o presente estudo tem por objetivo ouvir e compreender como usuários de uma unidade de atenção básica à saúde, portadores de DCME, percebem a dor e quais as crenças e os significados atribuídos às estratégias de cuidados em saúde desenvolvidas. 


\section{Metodologia}

\subsection{Desenho do estudo}

O presente estudo é uma pesquisa-ação com abordagem qualitativa, que empregou a técnica de grupos focais para investigação e análise das percepções, crenças e tomadas de decisão para o autogerenciamento da dor de portadores de DCME, usuários cadastrados na única Unidade Básica de Saúde (UBS) de uma comunidade num município do Sertão do Pajeú em Pernambuco.

Segundo Minayo, Deslandes e Gomes (2002), a pesquisa qualitativa responde a questões particulares, ou seja, trabalha com o universo de significados, motivos, aspirações, crenças, valores e atitudes, o que corresponde a um espaço mais profundo das relações, dos processos e dos fenômenos que não podem ser reduzidos à operacionalização de variáveis. Logo, tendo como objeto de estudo a DCME, e sabendose que nem sempre é possível compreendê-la por meio de escalas de avaliação, este estudo analisou os discursos dos participantes, buscando acessar as dores não verbalizadas, as falas ditas através do silêncio e as trocas de olhares entre os sujeitos.

Para acessar esse universo fez-se uso da técnica do grupo focal que utiliza a interação grupal para produzir dados e insights que seriam dificilmente conseguidos fora do grupo. Adicionalmente, conserva o caráter de técnica de coleta de dados, adequado para investigações qualitativas (KIND, 2004).

O grupo de entrevistadores foi composto por discentes do curso de Bacharelado em Medicina e as entrevistas foram guiadas pelas orientadoras da pesquisa, uma enfermeira e uma fisioterapeuta, ambas professoras com títulos, respectivamente, de mestrado e doutorado. A enfermeira possui amplo conhecimento e experiência em pesquisas qualitativas e a fisioterapeuta, em dor crônica e distúrbios musculoesqueléticos. Além disso, a fisioterapeuta é portadora de DCME há mais de dez anos, fato que proporcionou uma interação mais empática com os participantes da pesquisa.

$\mathrm{O}$ relacionamento da equipe de pesquisa com os voluntários ocorreu durante as discussões dos grupos focais, momentos em que pesquisadores observavam e colhiam os relatos sobre dor crônica. Aos participantes foi explicado o objetivo e as atividades para a execução da pesquisa, como o dia, a hora e o local para a reunião dos grupos focais. As orientadoras do projeto apresentaram toda a equipe de pesquisa aos participantes. 


\subsection{População e amostra}

A população-alvo foi constituída por todos os usuários cadastrados na USF, totalizando aproximadamente 4.400 pessoas. A amostragem foi do tipo não probabilística do tipo consecutiva, ou seja, resultou do arrolamento de toda a população acessível em um período suficiente para incluir mudanças relevantes à questão da pesquisa (HULLEY et al., 2008). O convite para participar da pesquisa foi feito pessoalmente pela equipe de entrevistadores durante as visitas domiciliares realizadas em conjunto com os Agentes Comunitários de Saúde (ACS). Esta etapa da pesquisa, juntamente com a triagem para elegibilidade e as intervenções em grupo, ocorreu ao longo dos dois semestres de 2019.

Considerando a temática abordada e os procedimentos de pesquisa, estabeleceuse um número máximo de 5 a 7 participantes por grupo focal, de acordo com os critérios de Debus (1995), para quem determinadas questões exigem minigrupos para que sejam abordadas em profundidade (KIND, 2004; DEBUS, 1995).

A amostra inicial foi composta por 54 participantes, que preencheram os critérios de elegibilidade e aceitaram participar livremente da pesquisa e dos grupos focais. Os pseudônimos mencionados nessa pesquisa, foram inspirados nos astros do Sistema Solar.

\subsection{Critérios de elegibilidade}

Os critérios de inclusão foram pacientes da UBS, ambos os sexos e idade entre 18 e 59 anos; presença de DCME por um período superior a 3 meses, categorizada de 3 a 7 na Escala Analógica Visual (EAV) (HUSKISSON, 1974); funções cognitivas preservadas, além de boa compreensão da língua portuguesa escrita e falada.

Foram excluídos aqueles com dor categorizada como 1 a 2 (floor effect) ou 8 a 10 (ceiling effect) por meio da EAV; diagnóstico de patologias do sistema nervoso com comprometimento motor ou cognitivo; histórico de câncer, traumatismos recentes, doenças infectocontagiosas ou acidentes vasculares súbitos.

\subsection{Recrutamento, procedimentos de coleta de dados e instrumentos de pesquisa}

Os participantes foram triados quanto aos critérios de elegibilidade, a partir de visitas domiciliares conduzidas pelos ACS e acompanhadas pela equipe de pesquisa. Os procedimentos de coleta de dados foram realizados no centro de convivência situado em prédio anexo ao da UBS, estando presente no momento das sessões de grupo focal os 
participantes, a equipe de pesquisa e, em alguns momentos, os filhos dos entrevistados, em geral, crianças.

Os instrumentos de triagem utilizados foram a ficha de cadastro individual e o questionário autorreferido de condições/situações de saúde do Sistema Único de Saúde (SUS), além da EAV. Os dois primeiros são utilizados rotineiramente pelos ACS para cadastro e rastreio das condições de saúde dos usuários atendidos pela equipe de Atenção Básica. Tais instrumentos foram utilizados para delinear e compreender o perfil amostral, uma vez que contêm identificação dos usuários, situações sociodemográficas, condições/situações de saúde gerais, além de conter informações sobre cidadãos em situação de rua.

A percepção da intensidade dolorosa foi avaliada por meio da EAV que consiste em uma escala unidimensional e linear, com pontuação numérica associada à escala de faces, variando de 0 (ausência de dor) a 10 (pior dor imaginável). Escores $\leq 3.4$ foram mais bem descritos como dor leve, 3.5-7.4 como dor moderada e $\geq 7.5$ como dor intensa para pacientes com dor musculoesquelética crônica (BOONSTRA et al., 2014).

Considera-se grupo focal como um procedimento de coleta de dados no qual o pesquisador tem a possibilidade de ouvir vários sujeitos ao mesmo tempo, observando as interações características do processo grupal. Tal técnica visa obter uma variedade de informações, sentimentos, experiências e representações acerca de um determinado tema (KIND, 2004). Dessa forma, após as entrevistas de avaliação, foram realizados 6 encontros, sendo um por semana, com duração máxima de 60 minutos, seguidos das entrevistas de reavaliação. Todos os encontros foram gravados em áudio (DEBUS, 1995).

A equipe de pesquisa distribuiu as funções de observador, moderador e relator entre seus integrantes, desempenhando a análise da rede de interações presentes durante o processo grupal, restringindo-se ao registro de linguagem, atitudes, preocupações e ordem das respostas. De acordo com Kind (2004), o observador é responsável, principalmente, pela análise das interações presentes no decorrer da discussão no grupo focal. Segundo Minayo (2009), o moderador apresenta o tema que será discutido e esclarece aos participantes o motivo da pesquisa; e o relator é aquele que anota os detalhes da reunião, podendo gravar ou filmar o processo de discussão, com o consentimento dos participantes.

As discussões no grupo ocorreram simultaneamente às intervenções. As perguntas disparadoras foram:

- "O que é dor/ De onde vem essa dor?". 
- "O que você sente quando sente dor/ Quais as sensações?".

- "Por que você acha que sente dor/ Qual o motivo de sua dor?".

- “O que faz para a dor passar/ Como você cuida de sua dor?”.

- "Você tem algum medo em relação a essa dor?".

- “Como você acredita que seus familiares veem sua dor?”.

- "Como você acredita que os profissionais de saúde veem sua dor?".

Durante a execução dos grupos focais, a equipe de pesquisa realizou notas de campo, destacando observações sobre os relatos e a linguagem não verbal dos voluntários. $\mathrm{O}$ acolhimento dos participantes e as rodas de conversa, cujos moderadores foram os profissionais de saúde que integram a equipe deste projeto de pesquisa, foram conduzidas dentro dos princípios da educação em dor baseada em neurociências. De acordo com Bonifácio (2019, p.18), "a neurociência contribui para a compressão do quadro de dor nos aspectos fisiológicos, cognitivos e comportamentais e suas relações com o processo de doença". Somado a isso, alcançar a reconceituação da dor pode aumentar a capacidade dos pacientes de lidar com sua condição álgica (WATSON et al., 2019). As sessões tiveram a seguinte sequência:

Quadro 1: Sequência de sessões

\begin{tabular}{|c|c|}
\hline Sessões & Atividades realizadas \\
\hline \multirow{3}{*}{ Sessão 1} & $\begin{array}{l}\text { Acolhimento dos participantes e apresentação do projeto de pesquisa e dos } \\
\text { objetivos das intervenções; }\end{array}$ \\
\hline & Roda de conversa: Entendendo a dor e o ciclo da dor crônica; \\
\hline & Discussão sobre conceitos e crenças a respeito da dor. \\
\hline \multirow[b]{2}{*}{ Sessão 2} & $\begin{array}{l}\text { Roda de conversa: Um convite à reflexão sobre a medicalização da dor e o } \\
\text { pensamento negativo; }\end{array}$ \\
\hline & $\begin{array}{l}\text { Discussão sobre os efeitos estimulantes das campanhas publicitárias para a } \\
\text { prática da automedicação. }\end{array}$ \\
\hline \multirow{3}{*}{ Sessão 3} & $\begin{array}{l}\text { Roda de conversa: Planejamento semanal de modo racional das atividades } \\
\text { domésticas e físicas; }\end{array}$ \\
\hline & Discussão e compartilhamento de estratégias de planejamento das atividades; \\
\hline & $\begin{array}{l}\text { Estímulo à mobilidade: Aprendizado de técnicas de relaxamento com } \\
\text { exercícios respiratórios. }\end{array}$ \\
\hline \multirow{3}{*}{ Sessão 4} & Roda de conversa: Relação da dor crônica com a qualidade do sono; \\
\hline & $\begin{array}{l}\text { Discussão e compartilhamento de estratégias para melhorar a qualidade do } \\
\text { sono; }\end{array}$ \\
\hline & $\begin{array}{l}\text { Estímulo à mobilidade: Realização de exercícios de relaxamento e } \\
\text { respiratórios. }\end{array}$ \\
\hline \multirow[b]{2}{*}{ Sessão 5} & $\begin{array}{l}\text { Roda de conversa: Atividades prazerosas e a influência da dor crônica em } \\
\text { suas realizações; }\end{array}$ \\
\hline & $\begin{array}{l}\text { Estímulo à mobilidade: Realização de exercícios de relaxamento e } \\
\text { respiratórios. }\end{array}$ \\
\hline
\end{tabular}




\begin{tabular}{|l|l|}
\hline \multirow{2}{*}{ Sessão 6} & $\begin{array}{l}\text { Roda de conversa: Percepção dos voluntários sobre as intervenções e sobre a } \\
\text { dor crônica; }\end{array}$ \\
\cline { 2 - 3 } & $\begin{array}{l}\text { Estímulo à mobilidade. Realização de uma roda de ciranda com os } \\
\text { participantes. }\end{array}$ \\
\hline
\end{tabular}

Fonte: Dados dos autores, 2019.

\subsection{Análise de dados}

Após transcrição do material gravado, a análise de dados e obtenção dos resultados foi realizada pelo método de Análise de Conteúdo de Bardin (BARDIN, 1977). Numa primeira fase, o material foi analisado para a escolha das Unidades de Registro (UR), sendo o tema DCME escolhido como UR; após isso, foram elaboradas as hipóteses provisórias e a organização do material que compõe o corpus da pesquisa. Nesta etapa seguiram-se as regras da exaustividade, representatividade, homogeneidade, exclusividade e pertinência, para, a partir disso, serem incluídos os conteúdos dos quais se originaram as categorias apropriadas. Em seguida, ocorreu a exploração dos materiais organizados na etapa anterior; nela incide a enunciação, codificação e classificação do corpus. Para esta pesquisa foi utilizada a técnica de Análise Categorial Temática (BARDIN, 1977).

As categorias resultantes da análise foram: "Percebendo a dor crônica e os pensamentos associados", cujas subcategorias são pessimismo e conformismo; "A dor 'mexe no psicológico' mesmo e o que costuma ser feito?”, tendo a medicalização como subgrupo; "O ponto de virada: participação no projeto de educação em dor crônica", sendo a interpretação dos relatos dividida entre metanoia, autocuidado e superação.

O presente estudo seguiu as diretrizes e normas que regulamentam as pesquisas envolvendo seres humanos, contidos na resolução 466, de 12 de dezembro de 2012, do Conselho Nacional de Saúde (CNS); tendo sido aprovado por Comitê de Ética em Pesquisa e registrado na Plataforma Brasil sob parecer no 3.041.726.

\section{Resultados e discussão}

Dentre os 54 participantes da amostra inicial, 24 compareceram a pelo menos uma sessão de grupo focal. O perfil amostral caracterizou-se pela predominância do sexo feminino $(91,67 \%)$, cor autorreferida parda $(70,83 \%)$, nível de escolaridade até o Ensino Fundamental $(45,83 \%)$, desemprego $(29,17 \%)$ ou ausência de trabalho formal $(37,5 \%)$. 
A DCME consiste no principal fator de procura por atendimento médico no setor primário de saúde (GIOVANINI, 2019). Segundo Mallon et al. (2019), indivíduos entre 40 e 59 anos apresentam um aumento na prevalência de DCME entre 51 e 67\%. O sexo feminino parece ser mais predisposto à DCME, devido a associação de diversos fatores psicossociais e, ainda, à participação dos hormônios gonadais na modulação neuroimunológica da dor (CASTRO et al., 2019). Paralelamente a isso, o baixo nível de escolaridade impacta diretamente na renda familiar e no enfrentamento da dor, além de favorecer um inadequado autogerenciamento terapêutico (KÖPPEN, 2018).

Após a realização das sessões, 9 participantes interagiram de modo mais significativo, resultando na amostra final e nos relatos explicitados neste artigo. Todos os participantes são portadores de DCME, com mediana da dor percebida, obtida pela EAV, de 7 [6-7]. Foram atribuídos os pseudônimos Vênus, Marte, Mercúrio, Saturno, Júpiter, Estrela, Lua, Terra e Sol, sendo o último participante o que compareceu e interagiu em quase todos os encontros realizados no decorrer da coleta - esteve presente em cinco das seis sessões.

Nota-se que houve uma evasão significativa dentre aqueles que aceitaram participar da pesquisa. Dentre os motivos discutidos pelos pesquisadores há o não reconhecimento da educação em dor como intervenção que tenha real efeito sobre a vivência álgica, uma vez que a maioria dos voluntários atribui ao 'remédio' a melhor forma de tratamento. O fenômeno da não adesão ao tratamento da dor crônica foi abordado no estudo de Kurita e Pimenta (2003); segundo as autoras, portadores de dores crônicas têm um longo histórico de sofrimento psíquico e de experiências negativas com resultados insatisfatórios de tratamentos anteriores. Esse contexto vivencial favorece a não adesão e a evasão, prolongam a dor e o sofrimento, ocasionam prejuízos funcionais e deterioram a qualidade de vida.

A análise do material ocorreu a partir das anotações das falas e expressões dos participantes pelos pesquisadores durante as sessões e da transcrição do material gravado, separando, após a obtenção dos dados, os relatos em diferentes categorias de acordo com o sentido e contexto do que foi explicitado. A partir dos relatos dos integrantes, agrupouse as falas em três grandes categorias que englobam: percebendo a dor crônica e os pensamentos associados; a dor "mexe no psicológico" mesmo e o que costuma ser feito; e o impacto da participação no projeto de educação em dor na mudança de pensamento e de comportamento. 
Inseridas nesse contexto, foram estabelecidas seis subcategorias de análise: pessimismo, conformismo, medicalização, metanoia, autocuidado e, por fim, superação. Dessa forma foi possível observar os sentimentos expressos através da verbalização ou não, bem como sua compreensão, mudanças de pensamentos e comportamentos diante da dor.

\subsection{Percebendo a dor crônica e os pensamentos associados}

A percepção dolorosa é uma experiência de cunho individual e subjetivo, com diferentes níveis de tolerância. Por conseguinte, vários fatores influenciam a compreensão desse fenômeno, dentre os quais figuram aspectos socioeconômicos, emocionais, comportamentais e cognitivos (PASSARELLI, 2017). A experiência dolorosa é um evento único e pessoal, e como tal foi expresso de maneira particular por cada participante:

"Todo movimento que eu faço eu lembro da dor" (Sol).

"A pessoa sente uma dor e pensa que a dor dela é maior que a das outras" (Vênus).

"Tem horas que você está com uma dor nos braços que não consegue botar uma camisa" (Estrela).

"[A dor] começa no lugar, fisicamente, e fica na mente" (Sol).

Nessa perspectiva, Santos, Giacomin e Firmo (2015) salientam que cada grupo social tem uma linguagem própria, pela qual as pessoas enfermas demonstram aos demais os sentidos do que as fazem sofrer. Atrelado a isso, o psiquiatra Dejours (2006) afirma que a negação é uma das primeiras estratégias de defesa para lidar com o sofrimento psíquico. Dessa forma, a utilização da negação como um instrumento de autodefesa para conviver com a dor crônica, pode ser percebida através da fala do participante Sol:

“Mas não interfere no meu humor, nem a dor, nem o sono" (Sol).

No decorrer da primeira sessão, questionou-se sobre a percepção e/ou conceituação da dor, tendo sido feita a pergunta "O que é dor?". As falas dos participantes demonstraram sempre uma perspectiva negativa do fenômeno:

"É um tormento" (Mercúrio).

"Ter dor é triste” (Saturno).

"É um incômodo" (Marte).

"É o limite" (Júpiter). 
Nesse momento, quando foi exposto que a dor em si é um mecanismo de alerta para o organismo, alguns dos participantes não entenderam como ela, que traz mais impactos negativos do que positivos em suas vidas, poderia estar relacionada com a proteção do corpo. Marquez (2011) aborda a existência desse mecanismo e o quanto ele pode se tornar disfuncional para o organismo, ao afirmar que as dores agudas são sinais de alerta de grande importância para a sobrevivência; ao passo que é necessário compreender que as dores crônicas não possuem finalidade biológica e, algumas vezes, nem nexo causal, constituindo-se como uma verdadeira doença.

Diante da oportunidade de falar sobre a sua percepção do fenômeno doloroso, os pacientes relataram suas reais condições e necessidades, reconhecimentos que podem trazer significativos benefícios para a evolução no tratamento. Percebe-se uma complexa interação entre a dor e as alterações emocionais, uma vez que ela é, ao mesmo tempo, causadora e resultante dessas modificações (SILVA; ROCHA; VANDENBERGHE, 2010).

\subsubsection{Pessimismo}

No processo grupal, também foi possível observar diferentes sentidos conferidos à dor: o pessimismo, o medo e a incerteza quanto ao futuro. De acordo com Palmeira (2015) nos estudos que trazem o significado da dor, são fortemente discutidas a dificuldade de compartilhar a experiência álgica e a deslegitimação social, trazendo, ainda, a dimensão da individualização da dor, a ideia de solidão, isolamento, bem como, as incertezas com o futuro. Realidade presente na fala de um dos participantes:

"Eu tenho muito medo que a dor volte" (Sol).

"Depois dessa dor, tudo mudou” (Sol).

Tais relatos corroboram com Dionísio, Salermo e Padilha (2020), quando afirmam que existem fortes evidências de que o medo relacionado à dor esteja muito mais associado à incapacidade percebida e ao desempenho reduzido, do que propriamente dito à magnitude da dor. Assim sendo, existe alta probabilidade de prognóstico reservado a desfavorável para a reabilitação de pacientes com crenças distorcidas em relação à dor (MILLS; NICOLSON; SMITH, 2019).

“Esse povo pensa negativo, diz uma coisa e a dor já aumenta. Às vezes é uma coisa bem simples e aumenta só com o pensamento negativo das outras pessoas" (Vênus). 


\subsubsection{Conformismo}

No que concerne às percepções dos distúrbios do sono relacionados à dor, observou-se um sentimento fortemente expresso pelos pacientes - o conformismo, conjugado a sensações de abatimento, desamparo e derrotismo:

"Só durmo com remédio" (Sol).

"De todo jeito tem que passar remédio... e a gente toma” (Vênus).

"Nem todos os dias são bons [em relação ao sono]" (Marte).

"Eu coloquei na cabeça que ia morrer e ia deixar minha filha" (Estrela).

"Não tenho equilíbrio de jeito nenhum" (Sol).

Segundo Marquez (2011), existe uma relação entre a dor crônica e a morte, sendo o processo de morte caracterizado pelas seguintes etapas: negação, raiva, depressão, aceitação da incapacidade e o próprio óbito. Dessa forma, os portadores de dor crônica passariam por todas essas fases, exceto pela última (MARQUEZ, 2011). Assim, quando o paciente chega na fase de incapacidade é fundamental a realização de uma abordagem mais realística no tratamento, visando o despertar para a importância da aceitação de si mesmo enquanto portador de dor crônica. Tal atitude é o ponto de partida para tomada de decisões objetivando o autogerenciamento da dor. Aceitar o próprio corpo e respeitar as suas limitações, possibilita aos portadores de dor crônica encontrar caminhos alternativos para lidar com os problemas álgicos associados aos eventos do cotidiano (JESUS; SVENTNICKAS; VIEIRA, 2019).

\subsection{A dor "mexe no psicológico" mesmo e o que costuma ser feito?}

No presente estudo, percebe-se que, ao tentarem descrever suas dores, os participantes apresentam relatos carregados de emoções negativas que demonstram a amplificação da dor:

"Eu já senti muita dor. Mas igual a essa.... Mexeu comigo. Mexeu com meu psicológico. Mudou realmente a minha vida, meus planos de vida, minha mente" (Sol);

"Minha autoestima mudou, eu não conseguia mais sorrir. Veio depressão, ansiedade..." (Sol).

Existe um emaranhado intrínseco entre emoção, cognição e dinâmicas interpessoais com os processos corpóreos da dor. A interação entre a fisiologia da dor e o sofrimento subjetivo do indivíduo esclarece como pode ser confuso tentar distinguir a dor com causa física e a dor com causa subjetiva. Ao levar a dor relatada pelo paciente 
para o nível imaginário ou "psicológico", ao afirmar que essa dor não é física, o profissional de saúde subestima o quanto esse sofrimento está marcado no corpo físico. Essa dicotomia entre dor física e dor emocional é artificial, desqualifica a vivência do paciente e gera frustração e descrença na terapêutica (SILVA; ROCHA; VANDENBERGHE, 2010).

Portadores de DCME que apresentam sintomas depressivos e crenças disfuncionais sobre a dor apresentam redução da motivação em participar de atividades sociais, distúrbios do sono, criação de situações de dependência e redução da sensação de bem-estar (MALLON et al., 2019). A relação entre dor crônica e distúrbios do sono é bidirecional, ou seja, a dor causa um sono insatisfatório, e o sono de má qualidade amplifica a percepção dolorosa, prolongando-a (MILLS; NICOLSON; SMITH, 2019). Tal questão pode ser observada nos seguintes relatos:

"Você quer dormir a todo custo. Mas é difícil..." (Sol).

"Essa dor sempre aparece de noite, eu não consigo dormir. Tem noite que é pior que dia" (Lua).

\subsubsection{Medicalização}

No intuito de reduzir o impacto dos distúrbios do sono associados à dor crônica, muitos pacientes acabam por recorrer ao uso de sedativos. Segundo Jank et al. (2017), cerca de $10 \%$ a $20 \%$ dos portadores de dor crônica com distúrbios do sono fazem uso de fármacos para dormir; contudo, nem sempre o fazem com prescrição médica. Outrossim, além de fazer uso de fármacos por conta própria, acreditam que a medicação é a única solução para o problema:

"Há seis meses que eu só durmo com remédio. Antes dormia de 21 horas, depois que veio essa dor, eu passei seis dias e seis noites sem dormir. Daí para cá, só com remédio" (Sol).

"Sinto falta de quando eu dormia a noite todinha. Hoje é como se eu fosse anestesiada [pelo efeito de sedativos]. Simplesmente você dorme. Meu corpo não descansa com o remédio. Sinto falta de ter uma vida saudável” (Sol).

"Quando chega a noite, é a hora que a gente mais precisa desse remédio. É muito difícil resistir. É muito cômodo ter certeza que eu vou conseguir dormir” (Sol).

“O remédio vicia muito rápido. Seis meses só. Toda noite é uma briga para não tomar, mas eu fico com medo da dor, porque se eu não tomar eu vou ficar com ela" (Sol). 
"Não importa o dinheiro que é gasto, se comprar o remédio e a dor passar (Estrela)".

"Daí eles [médicos] nos falam que se tomar o remédio, [a dor] passa (Estrela); Hoje mesmo fui na farmácia, comprei um remédio e tomei. Não sei nem o nome do remédio. Só tomei e já estou aqui” (Júpiter).

Adicionalmente, a automedicação também é prática frequente entre pacientes que anseiam reduzir os impactos que a persistência da dor causa na capacidade funcional e na vida social; a exemplo da paciente Júpiter, que na tentativa de se incluir socialmente, em uma das sessões revelou que se automedicou para conseguir estar presente naquele dia. Nesse sentido, como abordam Meyer, Denis e Berquin (2018), a constância da percepção dolorosa é capaz de afetar a manutenção de uma vida social saudável, podendo causar danos à saúde mental.

\subsection{O ponto de virada: participação no projeto educação em dor crônica}

Na intervenção terapêutica dos distúrbios consequentes à DCME, há evidências convincentes de que uma estratégia educacional abordando a neurofisiologia e a neurobiologia da dor pode ter um efeito positivo tanto nos aspectos físicos como a incapacidade, quanto no medo e nas crenças disfuncionais sobre o fenômeno doloroso (LOUW et al., 2011).

A dor é percebida não apenas pela experiência pessoal, mas também indiretamente pela comunicação e interação social (SILVA et al., 2019). Compreender as dificuldades físicas e emocionais relacionadas à dor, enfrentadas pelos pacientes, apenas foi possível diante da análise de seus relatos por meio da interação obtida no grupo focal. Além disso, os relatos demonstram algumas modificações nas crenças e comportamentos dos participantes, mediante as orientações dadas e os exercícios de relaxamento e respiração instruídos durante as rodas de conversa de educação em dor. Tais mudanças podem ser observadas em algumas falas:

"O exercício da respiração funciona quase que 100\%” (Sol).

"O jeito mais dificil é o que funciona melhor” (Sol).

"Depois que aprendi a respiração [exercícios de respiração], estou bem melhor" (Terra).

Essa perspectiva de "ponto de virada" também foi observada em estudo metodologicamente similar, realizado com 8 pacientes numa UBS na região Sul do Brasil, 
no qual a compreensão das limitações dos movimentos corporais devido à DCME e o sentimento de estar recebendo apoio foram percebidos, pelos pacientes, como fatores fundamentais para gerar motivação e impactar no estado geral de saúde e autonomia dos participantes (JESUS; SVENTNICKAS; VIEIRA, 2019). Os efeitos positivos da educação em dor na percepção dolorosa, incapacidade e crenças disfuncionais podem permitir que os pacientes apliquem essa nova visão de seu estado de dor, reavaliando sua capacidade de se mover.

\subsubsection{Metanoia}

Metanoia significa a ação de mudar de ideia ou pensamento, deixar de acreditar em um determinado evento ou fenômeno para vivenciar uma nova forma de conduzir a vida. Nesta óptica, foi possível observar que a participação nas sessões grupais, conduzidas dentro dos princípios da educação em dor (LOUW et al., 2011), estimulou mudanças nas atitudes e no modo de gerenciar a dor em alguns participantes; trazendo para si o controle e a autorresponsabilidade sobre sua realidade, possibilitando uma maior aceitação dos ensinamentos transmitidos nas intervenções, como a prática de exercícios respiratórios e de relaxamento. Para Araújo (2020), aprender a autogerenciar a dor traz para o paciente um maior respeito às limitações do corpo, a capacidade de autoconhecimento, de autocuidado e, ainda, de se adaptar. Como pode ser observado nos seguintes relatos:

“Quem tá no controle agora sou eu” (Sol).

"Vou fazer em casa [os exercícios de respiração]. Dói, mas é bom. Bom, gostei. Vou fazer" (Sol).

Com a diminuição da ameaça de lesão tissular adicional e uma percepção recémadquirida de que a dor pode ser causada pela sensibilidade neural em vez da lesão do tecido, os pacientes podem ser capazes de mover-se ativamente reduzindo a sensação de dependência funcional e restaurando a autonomia (LOUW et al., 2011).

A adoção de estratégias ativas no enfrentamento da dor crônica, como o exercício, diminui a procura por consultas médicas, diminuindo também o nível de incapacidade devido à dor em comparação aos que adotam estratégias passivas, como o descanso e a medicação (MILLS; NICOLSON; SMITH, 2019). 


\subsubsection{Autocuidado}

Segundo Jesus, Sventnisckas e Vieira (2019), compreender e refletir o processo saúde-doença auxilia no estabelecimento de maior autonomia e capacidade de autocuidado. Ao refletirem sobre os fatores envolvidos na persistência da dor, os participantes desta pesquisa perceberam sua capacidade de executar ações que minimizem ou evitem a dor, tornando-se mais comprometidos com a própria saúde e o autocuidado. Tais fatos podem ser observados nos relatos:

"Só penso em mim. Cuidar da minha saúde” (Sol).

"O primeiro exercício, que inspirou pelo nariz, dá mais tontura. Quando respira e solta pela boca é melhor, mas também faço o primeiro [exercício]" (Sol).

"É o querer. E eu quero muito ter saúde” (Sol).

"Faço os exercícios três vezes ao dia. Igual remédio controlado. Faço todos os dias" (Sol).

"Eu tô lutando contra a ansiedade. Mas eu parei de tomar remédio. Porque eu quero o controle da minha vida" (Sol).

"Eu tô tentando resgatar quem eu era" (Sol).

Além disso, o participante Sol afirma ainda que está sempre optando por não executar atividades exaustivas quando sente dor e que tem enfrentado seus medos na hora de dormir, pois frequentemente tem pesadelos. A modificação do padrão comportamental reforça as atitudes de autocuidado e melhora a sensação de autonomia e bem-estar (SANTOS; LUNA; COUTINHO, 2019).

\subsubsection{Superação}

Conhecer e aceitar o próprio corpo, respeitando suas limitações, possibilitou aos participantes encontrarem caminhos alternativos para lidar com tensões musculares, restrições da amplitude de movimento, queixas álgicas associadas a eventos do cotidiano e o medo do movimento. A percepção e conscientização corporal, bem como o entendimento da dor como um processo sobre o qual se pode interferir com estratégias de cuidado, contribui para que o paciente se sinta mais confiante na autogestão e superação de seu problema (JESUS; SVENTNISCKAS; VIEIRA, 2019). Isso foi observado, principalmente, nos relatos do participante Sol:

"Seis meses com a bengala. Eu sou persistente. Depois que eu aprendi os exercícios aqui, eu fiz todos os dias. Faço todo dia. E agora tô livre [da bengala]” (Sol). 
"Pela primeira vez eu consegui dormir, dormir mesmo" (Sol).

"Foi a prova [fortes dores] mais difícil que eu já passei na vida” (Sol).

"Eu tô resistindo tanto para não tomar injeção. Eu tô com três injeções guardadas, faz três meses porque é uma por mês. Eu não quero ser dependente de nenhuma droga" (Sol).

"Eu saí de 4 remédios (Sol); Eu deixo ela lá [a bengala], só pra ela ver que quem manda sou eu” (Sol).

Diante dos relatos entende-se que o enfrentamento ativo do fenômeno dor e sua persistência contribui para uma efetiva superação (CHOU et al., 2017). O participante Sol, que antes possuía atividades limitadas, depois das intervenções, mesmo com muito esforço, começa a se dispor a adotar medidas de autogerenciamento que antes não eram estabelecidas pelo desconhecimento, pelas crenças negativas e pela negação da dor. Paralelamente, outro ponto de igual importância, e relatado por vários participantes, foi a redução do uso de medicações, decorrente da diminuição das dores, atenuando a dependência dos fármacos (MALFLIET et al., 2017).

Dentre os principais aspectos positivos dessa pesquisa, está o fato de se tratar de uma abordagem qualitativa, ainda pouco explorada para abordar a dor persistente. Tal desenho metodológico favorece uma análise do fenômeno doloroso sob uma perspectiva mais humanizada, possível por meio dos grupos focais e da escuta atenta da fala do portador de dor crônica. Para Vieira, Bartz e Jornada (2017, p.312), "são escassos os estudos qualitativos, que exploram as questões mais subjetivas relacionadas à dor e as percepções dos seus portadores”.

A limitação mais significante encontrada durante a pesquisa foi a pequena aderência dos participantes que iniciaram as sessões e não deram continuidade. Contudo, ressalta-se que, segundo Kind (2004), não há consenso sobre a quantidade ideal de participantes em grupos focais, enfatizando que a alta quantidade de participantes pode comprometer a qualidade da pesquisa pela dificuldade, por exemplo, de engajamento.

\section{Conclusão}

A característica multidimensional da dor foi explicitada nesta pesquisa, com a exposição das percepções e da subjetividade dos portadores de dor crônica diante da sua condição. Em geral, eles encaram a vivência álgica com dificuldade e, corriqueiramente, fazem uso da automedicação como forma de escape. No entanto, a alteração 
comportamental, proporcionada pelas sessões, por meio do enfrentamento ativo e do desenvolvimento de atitudes de autocuidado foram importantes para mudanças em vários aspectos da vida dessas pessoas.

A partir dos relatos analisados, a pesquisa demonstra que a educação em dor é um meio importante para os pacientes conhecerem os múltiplos aspectos que influenciam o fenômeno doloroso, modificando seus hábitos diários, adaptando-se diante da dor e não se limitando perante ela. Por fim, encontros pautados pela escuta atenta e pelo estímulo a uma corresponsabilidade no cuidado em saúde, contribuem, de maneira significativa, para uma maior autonomia, o que motiva os participantes a incorporarem novas estratégias para lidar com a persistência da dor.

\section{Referências}

AGUIAR G. P. S.; DUSSAN-SARRIA J. A. S.; SOUZA A. Alterações do sono em pacientes vivendo com vírus da imunodeficiência humana e dor crônica. BrJP, São Paulo, v. 2, n. 2, p. 123-131, apr-jun. 2019.

ARAÚJO S. C. S. C. A Gestão da dor crónica na pessoa com demência avançada: saberes e práticas dos profissionais de saúde de uma estrutura residencial. 2019. 115 f. Dissertação (Mestrado em cuidados paliativos) - Instituto Politécnico de Viana do Castelo, Viana do Castelo, Portugal, 2019.

ARAUJO, A. B. M. Narrativas de vida de mulheres com fibromialgia: autogerenciamento da dor crônica. 2020. 100 f. Dissertação (Mestrado em Enfermagem) - Faculdade de Enfermagem, Universidade do Estado do Rio de Janeiro, Rio de Janeiro, 2020.

BARDIN, L. Análise de Conteúdo. 2. ed. Lisboa: Edições 70, 1977.

BONIFÁCIO, L. M. R. A educação em dor para profissionais de saúde com princípios de neurociência. 2019. 46 f. Monografia (Graduação em Farmácia) - Universidade de Brasília, Brasília, 2019.

BOONSTRA, A. M. et al. Cut-off points for mild, moderate, and severe pain on the visual analogue scale for pain in patients with chronic musculoskeletal pain. Pain®, Washington, v. 155, n. 12, p. 2545-2550, dec. 2014.

BOSCAROL, G. T. et al. Avaliação do diagnóstico de enfermagem "dor aguda" em pacientes internados em uma clínica médico-cirúrgica. Rev Enferm Cent Oeste Min, Divinópolis, v. 9, n. e3312, p. 1-9. 2019. Avaliação do diagnóstico de enfermagem "dor aguda" em pacientes internados em uma clínica médico-cirúrgica

CASTRO, S. et al. Implementação de atendimento ambulatorial para dor crônica: resultados preliminares. Rev Bras Anestesiol, Campinas, v. 69, n. 3, p. 227-232, may-jun. 2019.

CHOU, R. et al. Nonpharmacologic Therapies for Low Back Pain: a systematic review for an american college of physicians clinical practice guideline. Annals Of Internal Medicine, [S.L.], v. 166, n. 7, p. 493-507, fev. 2017. 
DEBUS, M. El manual para excelencia en la investigacón mediante grupos focales.

Washington: Academia para el Desarrollo Educativo, 1995.

DEJOURS, C. A banalização da injustiça social. 6. ed. Rio de Janeiro: FGV, 2006.

DE PAULA PRUDENTE, M. et al. Tratamento da dor crônica na atenção primária à saúde. Brazilian Journal of Development, Curitiba, v. 6, n. 7, p. 49945-49962, jul. 2020.

DIONÍSIO G. H.; SALERMO V. Y.; PADILHA A. Sensibilização central e crenças entre pacientes com dores crônicas em uma unidade de atenção primária de saúde. BrJP, São Paulo, v.3, n.1, p. 42-47, jan-mar. 2020.

GIOVANINI, L. F. L. Capacitação médica individualizada em dor crônica musculoesquelética generalizada na atenção primária à saúde. 2019,77 p. Dissertação (Mestrado em Educação nas Profissões da Saúde) - Pontifícia Universidade Católica de São Paulo, Sorocaba, 2019.

HULLEY, S.B. et al. Delineando a pesquisa clínica: uma abordagem epidemiológica. 4. ed. Porto Alegre: Artmed; 2008.

HUSKISSON, E. C. Measurement of pain. Lancet, Inglaterra, v. 304, n. 7889, p. 1127-1131, nov. 1974.

JAMES, L. S. et al. et al. Global, regional, and national incidence, prevalence, and years lived with disability for 354 diseases and injuries for 195 countries and territories, 1990-2017: a systematic analysis for the global burden of disease study 2017. The Lancet, [S.L.], v. 392, n. 10159, p. 1789-1858, nov. 2018.

JANK, R. et al. Chronic pain and sleep disorders in primary care. Pain Res Treat, Londres, v. 2017, n. 9081802, p. 1-9, dec. 2017.

JESUS, J. D.; SVENTNISCKAS, S. P.; VIEIRA, A. Grupo de Promoção à Saúde: Ampliando o Cuidado em Saúde de Usuários com Dores Musculoesqueléticas Crônicas em Serviços de Atenção Básica. Mov, Porto Alegre, v. 25, n. e25074, p. 1-14. 2019.

KIND, L. Notas para o trabalho com a técnica de grupos focais. Psicol Rev, Belo Horizonte, v. 10 , n. 15 , p. 124-138, oct. 2004.

KÖPPEN, P. J. et al. Health literacy, pain intensity and pain perception in patients with chronic pain. Wien Klin Wochenschr, Vienna, v. 130, n. 1-2, p. 23-30, jan. 2018.

KURITA, G. P.; PIMENTA, C. A. de M. Adesão ao tratamento da dor crônica: estudo de variáveis demográficas, terapêuticas e psicossociais. Arquivos de neuro-psiquiatria, São Paulo, v. 61, n. 2-B, p. 416-425. 2003.

LOUW, A. et al. The effect of neuroscience education on pain, disability, anxiety, and stress in chronic musculoskeletal pain. Archives of physical medicine and rehabilitation, Reston, v. 92, n. 12, p. 2041-2056, dec. 2011.

MALFLIET, A et al. Applying contemporary neuroscience in exercise interventions for chronic spinal pain: treatment protocol. Braz J Phys Ther, São Paulo, v. 21, n. 5, p. 378-387, set. 2017. 
MALLON, T. et al. Lifestyle Aspects as A Predictor of Pain Among Oldest-Old Primary Care Patients - A Longitudinal Cohort Study. Clin Interv Aging, Londres, v. 14, p. 1881-1888, nov. 2019.

MARQUEZ, J. O. A dor e os seus aspectos multidimensionais. Cienc Cult, Campinas, v. 63, n.2, p. 28-32, abr. 2011.

MENDES, L. A. S. Conversando com a dor: rodas de conversa sobre a dor crônica. 2019. 47 f. Monografia (Especialização em formação de Educadores em Saúde) - Universidade Federal de Minas Gerais, Belo Horizonte, 2019.

MEYER C.; DENIS C. M.; BERQUIN A. D. Secondary prevention of chronic musculoskeletal pain: A systematic review of clinical trials. Ann Phys Rehabil Med, [S.L.], v. 61, n. 5, p. 323338, set. 2018.

MILLS, S. E. E.; NICOLSON, K. P.; SMITH, B. H. Chronic pain: a review of its epidemiology and associated factors in population-based studies. Br J Anaesth, [S.L.], v. 123 n. 2, p. e273e283, ago, 2019.

MINAYO, M. C. S. Construção de indicadores qualitativos para avaliação de mudanças. Rev Bras Educ Med, Brasília, v. 33, n. 1 Supl. 1, p. 83-91. 2009.

MINAYO M. C. S. DESLANDES S.F.; GOMES R. Pesquisa social: teoria, método e criatividade. 21. ed. Petrópolis: Vozes, 2002.

MORAIS, B. X. et al. Musculoskeletal pain in undergraduate health students: prevalence and associated factors. Revista da Escola de Enfermagem da USP, São Paulo, v. 53, n. e03444, p. 1-8. 2019.

MOURA, C. C. et al. Impactos da dor crônica na vida das pessoas e a assistência de enfermagem no processo. Avances En Enfermería, [S.L.], v. 35, n. 1, p. 53-63, jan. 2017.

PALMEIRA, A. T. Experiência de enfermidade em pessoas com dor crônica atendidas em um serviço especializado de saúde. 2015. 194 f. Tese (Doutorado em Saúde Pública) Instituto de Saúde Coletiva, Universidade Federal da Bahia, Salvador, 2015.

PASSARELLI, M. Dor crônica: elaboração de infográfico como ferramenta em educação de leigos. 2016. 63 f. Dissertação (Mestrado em Educação nas Profissões da Saúde) - Pontifícia Universidade Católica de São Paulo, Sorocaba, 2016.

RAJA, S. N. et al. The revised International Association for the Study of Pain definition of pain: concepts, challenges, and compromises. Pain, Washington, v. 161, n. 9, p. 1976-1982, set. 2020.

RODRIGUES, A. C. et al. Factors that influence the quality of life in neuropathic, musculoskeletal, and oncological pain. BrJP, São Paulo, v. 4, n. 1, p. 31-36, jan-mar. 2021.

SANTOS, W. J.; GIACOMIN K. C.; FIRMO J. O. Alteridade da dor nas práticas de Saúde Coletiva: implicações para a atenção à saúde de pessoas idosas. Cienc Saúde Colet, Rio de Janeiro, v. 20, n. 12, p. 3713-3721, dez. 2015. 
SANTOS, A. L.; LUNA, M. B.; COUTINHO, R. S. Influência da dor lombar inespecífica na cinesiofobia: uma revisão integrativa. Revista Eletrônica da Estácio Recife, Recife, v. 5, n. 1, p. 1-12, jul. 2019.

SEMERU, G. M.; HALIM, M. S. Acceptance versus catastrophizing in predicting quality of life in patients with chronic low back pain. The Korean Journal of Pain, Seoul, v. 32. n. 1. p. 2229, jan. 2019.

SILVA, D. S.; ROCHA E. P.; VANDENBERGHE, L. Tratamento psicológico em grupo para dor crônica. Temas Psicol, Ribeirão Preto, v. 18, n. 2, p. 335-343, dec. 2010.

SILVA, M. F. et al. Avaliação da dor e seus aspectos multidimensionais. Rev Interdiscip Pensam Cient, [S.L.], v. 5, n. 4, p. 774-785, mai. 2019.

SOUZA, F. A. E. F. et al. A dor desde a perspectiva do ciclo de vida: avaliação e medição através de métodos psicofísicos de estimação de categoria e magnitude. Rev Lat Am Enferm, Ribeirão Preto, v. 24, n. e2769, p. 1-9, ago. 2016.

SOUZA, J. B. DE et al. Prevalence of Chronic Pain. Treatments. Perception. and Interference on Life Activities: Brazilian Population-Based Survey. Pain Research and Management, [S.L.], v. 2017, p. 1-9, sep. 2017.

VASCONCELOS, F. H.; ARAÚJO, G. C. de. Prevalence of chronic pain in Brazil: a descriptive study. BrJP, São Paulo, v. 1, n. 2, p. 176-179, apr./jun. 2018.

VIEIRA, A.; BARTZ, P. T.; JORNADA, M. C. Repercussão do grupo da coluna sobre o cotidiano de mulheres que apresentam dores musculoesqueléticas crônicas. Cad Bras Ter Ocup, São Carlos, v. 25, n. 2, p. 305-314. 2017.

WATSON, J. A. et al. Pain neuroscience education for adults with chronic musculoskeletal pain: a mixed-methods systematic review and meta-analysis. The Journal of Pain, Washington, v. 20, n. 10, p. 1140. e1-1140. e22, oct. 2019.

Recebido em: 20 de abril de 2021.

Aceito em: 16 de julho de 2021. 